Mathematical Sciences And Applications E-Notes

Volume 3 No. 1 PP. 137-153 (2015) @ CMSAEN

\title{
NUMERICAL SOLUTION OF FUZZY DIFFERENTIAL EQUATIONS BY MILNE'S PREDICTOR-CORRECTOR METHOD
}

\author{
MINE AYLIN BAYRAK AND EMINE CAN \\ (Communicated by Xiao-Jun YANG)
}

\begin{abstract}
In this paper Milne's predictor-corrector method to solve the fuzzy first first-order initial value problem are investigated. Sufficiently conditions for stability and convergence of the proposed algorithm are also proved. Their applicability is illustrated by two examples.
\end{abstract}

\section{INTRODUCTION}

Fuzzy differential equation (FDE) models play a prominent role in a range of application areas, including population models [1], particle systems [2],[3],[4],[5], quantum optics and gravity [6], synchronize hyperchaotic systems [7], control chaotic systems $[8],[9]$, medicine $[10],[11],[12],[13]$, to bioinformatics and computational biology $[14],[15],[16],[17],[18],[19]$. The first step which included applicable definitions of fuzzy derivative and the fuzzy integral was followed by introducing FDE and establishing sufficient conditions for the existence of unique solutions to these equations $[20],[21],[22],[23],[24]$. Finally, numerical algorithms of calculating approximates to these solutions where designed. A theoretical research of fuzzy first-order initial value problem was given by Kaleva [20],[21],[22],[23],[24], [25] Seikkala [22], Quyang and $\mathrm{Wu}[26]$, Kloeden [21] and $\mathrm{Wu}$ [27]. Some applications of numerical methods such as the fuzzy Euler, Adams-Bashforth, Adams-Moulton, Nyström and predictor-corrector in FDE presented in [28],[29],[30],[31].

This paper is organized as follows:

In Section 2, some basic definitions are presented. Milne's predictor-corrector method for solving fuzzy differential equations is introduced and predictor-corrector algorithm is discussed in Section 3. Convergence and stability of the mentioned methods are proved in Section 4. Two examples are presented in Section 5, and finally conclusion is drawn.

Date: Received: January, 9, 2015, Accepted: January, 25, 2015.

2010 Mathematics Subject Classification. 34A12,65L05.

Key words and phrases. Fuzzy differential equations; Fuzzy initial value problem; Predictorcorrector method; Milne's method. 


\section{Preliminaries}

In this section, some basic definitions of ordinary differential equations (ODEs) and the necessary notation used in fuzzy calculus are introduced. We begin by defining the $q$-step method.

Definition 2.1. Consider the initial value problem

$$
y^{\prime}(t)=f(t, y(t)), y(0)=y_{0}
$$

where $f:[a, b] \times \mathbb{R}^{n} \rightarrow \mathbb{R}^{n}$. A $q$-step method for solving Eq. (2.1) is one whose difference equation for finding $y_{i+1}$ as approximation $y\left(t_{i+1}\right)$ at the mesh point $t_{i+1}$ can be represented by the following equation:

$$
y_{i+1}=\sum_{j=0}^{q-1} a_{q-j-1} y_{i-j}+h \sum_{j=0}^{q-1} b_{q-j} f\left(t_{i-j+1}, y_{i-j+1}\right)
$$

for $i=q-1, q, \ldots, N-1$ such that $a=t_{0} \leq t_{1} \leq, \ldots, \leq t_{N}=b, h=\frac{b-a}{n}=t_{i+1}-t_{i}$ and $a_{0}, a_{1}, \ldots, a_{q-1}, b_{0}, b_{1}, \ldots, b_{q}$ are constant with the starting values $y_{0}=\alpha_{0}, y_{1}=$ $\alpha_{1}, y_{2}=\alpha_{2}, \ldots, y_{q-1}=\alpha_{q-1}$.

When $b_{q}=0$, the method is known as explicit, since Eq.(2.2) gives $y_{i+1}$ explicit in terms of previously determined values. When $b_{q} \neq 0$, the method is known as implicit, since $y_{i+1}$ occurs on both sides of Eq.(2) and is specified only implicitly. The special case $n=2$ is known as Milne's predictor-corrector method:

Predictor $(\mathrm{P})$ : Adams-Basforth method of fourth order. The method requires the starting values $y_{i}, y_{i-1}, y_{i-2}$ and $y_{i-3}$.

$$
y_{i+1}^{(P)}=y_{i-3}+\frac{4 h}{3}\left(2 f_{i}-f_{i-1}+2 f_{i-2}\right)
$$

where $\quad i=3,4, \ldots, N-1$.

Corrector $(\mathrm{C})$ : Milne-Simpson's method of fourth order. The method requires the starting values $y_{i}, y_{i-1}$.

$$
y_{i+1}^{(C)}=y_{i-1}+\frac{h}{3}\left(f\left(t_{i+1}, y_{i+1}^{(P)}\right)+4 f_{i}+f_{i-1}\right)
$$

where $\quad i=3,4, \ldots, N-1$.

Thus, for Milne's predictor-corrector (PC) method the combination requires the starting values $y_{i}, y_{i-1}, y_{i-2}$ and $y_{i-3}$.

Definition 2.2. Associated with the difference equation

$$
\begin{gathered}
y_{i+1}=a_{q-1} y_{i}+a_{q-2} y_{i-1}+\ldots+a_{0} y_{i+1-q}+h F\left(t_{i}, h, y_{i+1}, y_{i}, \ldots, y_{i+1-q}\right), \\
y_{0}=\alpha_{0}, y_{1}=\alpha_{1}, \ldots, y_{q-1}=\alpha_{q-1} .
\end{gathered}
$$

the following, called the characteristic polynomial of the method is $P(\lambda)=\lambda^{q}-$ $a_{q-1} \lambda^{q-1}-a_{q-2} \lambda^{q-2}-\ldots-a_{1} \lambda-a_{0}$. If $\left|\lambda_{i}\right| \leq 1$ for each $i=1,2, \ldots, q$, and all roots with absolute value 1 are simple roots, then the difference method is said to satisfy the root condition. The next result is proved in [32].

Theorem 2.1. A multistep method of the form (2.2) is stable if and only if it satisfies the root condition.

Proof. See [32]. 
The remainder of this section is developed to introducing the notation we shall be using in this paper and some definitions. All our fuzzy set will be fuzzy subsets of the real numbers $\mathbb{R}$. We place a tilde over a symbol to denote a fuzzy set so $\tilde{\alpha_{1}}, \tilde{u_{1}}, \ldots$ all represent fuzzy subsets on $\mathbb{R}$.

In what follows, according to the basic fuzzy calculus [33], we use the so-called $\alpha$-level setting to define a fuzzy number or interval, a fuzzy number (or interval) $u$ is completely determined by any pair $u=(\underline{u}, \bar{u})$ of functions $\underline{u}, \bar{u}:[0,1] \rightarrow R$, defining the end point of the $\alpha$-level, satisfying the three conditions:

1. $\underline{u}: \alpha \rightarrow \underline{u}^{\alpha} \in \mathbb{R}$ is a bounded monotonic increasing (nondecreasing) left-continuous function $\forall \alpha \epsilon(0,1]$ and right-continuous for $\alpha=0$.

2. $\bar{u}: \alpha \rightarrow \bar{u}^{\alpha} \in \mathbb{R}$ is a bounded monotonic decreasing (nonincreasing) left-continuous function $\forall \alpha \epsilon(0,1]$ and right-continuous for $\alpha=0$.

3. $\underline{u}^{\alpha} \leq \bar{u}^{\alpha}, 0 \leq \alpha \leq 1$.

If $\underline{u}^{1}<\bar{u}^{1}$, we have a fuzzy interval and if $\underline{u}^{1}=\bar{u}^{1}$, we have a fuzzy number; for simplicity we refer to fuzzy numbers as intervals.

Let us denote by $\mathbb{E}$ the class of subsets of the real axis $u: \mathbb{R} \rightarrow[0,1]$ satisfying the following properties:

(i) $u$ is normal, i.e. $\exists \mathrm{x}_{0} \in \mathbb{R}$ with $u\left(x_{0}\right)=1$;

(ii) $u$ is convex fuzzy set

(i.e. $u(t x+(1-t) y) \geq \min \{u(x), u(y)\}, \forall t \in[0,1], x, y \in \mathbb{R})$;

(iii) $u$ is upper semicontinuous on $\mathbb{R}$;

(iv) $\{x \in \mathbb{R} ; u(x)>0\}$ is compact, where $\bar{A}$ denote the clossure of $A$.

Then $\mathbb{E}$ is called the space of fuzzy numbers [34]. Meaning if $v \epsilon \mathbb{E}$ then the $\alpha$-level set

$[v]^{\alpha}=\{s \mid v(s) \geq \alpha\}, 0<\alpha \leq 1$,

is a closed bounded interval which we denote by

$[v]^{\alpha}=\left[\underline{v}^{\alpha}, \bar{v}^{\alpha}\right]$.

Let $I$ be a real interval. A mapping $y: I \rightarrow \mathbb{E}$ is called a fuzzy process and its $\alpha$-level set is denoted by $[y(t)]^{\alpha}=\left[\underline{y}^{\alpha}(t), \bar{y}^{\alpha}(t)\right], t \epsilon I, \alpha \epsilon(0,1]$.

A triangular fuzzy number $N$ is defined by an ordered triple $\left(x^{l}, x^{c}, x^{r}\right) \epsilon \mathbb{R}^{3}$ with $x^{l} \leq x^{c} \leq x^{r}$ where the graph of $N(s)$ is a triangle with base on the interval $\left[x^{l}, x^{r}\right]$ and vertex at $s=x^{c}$. An $\alpha$-level of $N$ is always a closed, bounded interval. We write $N=\left(x^{l}, x^{c}, x^{r}\right)$, then

$$
[N]^{\alpha}=\left[x^{c}-(1-\alpha)\left(x^{c}-x^{l}\right), x^{c}+(1-\alpha)\left(x^{r}-x^{c}\right)\right]
$$

for any $0 \leq \alpha \leq 1$.

Definition 2.3. Let $D: \mathbb{E} \times \mathbb{E} \rightarrow \mathbb{R}_{+} \cup\{0\}$. The Hausdorff distance between fuzzy numbers, where $[u]_{\alpha}=\left[\underline{u}^{\alpha}, \bar{u}^{\alpha}\right],[v]_{\alpha}=\left[\underline{v}^{\alpha}, \bar{v}^{\alpha}\right]$ is defined by

$$
D(u, v)=\sup _{\alpha \in[0,1]} \max \left\{\left|\underline{u}^{\alpha}-\underline{v}^{\alpha}\right|,\left|\bar{u}^{\alpha}-\bar{v}^{\alpha}\right|\right\}
$$

and $(\mathbb{E}, D)$ is a complete metric space.

Definition 2.4. A mapping $F: T \rightarrow \mathbb{E}$ is Hukuhara differentiable at $t_{0} \epsilon T \subseteq \mathbb{R}$ if for some $h_{0}>0$ the Hukuhara difference

$F\left(t_{0}+\Delta t\right) \sim_{h} F\left(t_{0}\right), F\left(t_{0}\right) \sim_{h} F\left(t_{0}-\Delta t\right)$, exist in $\mathbb{E}$ for all $0<\Delta t<h_{0}$ and if 
there exist an $F^{\prime}\left(t_{0}\right) \epsilon \mathbb{E}$ such that

$$
\lim _{\Delta t \rightarrow 0^{+}} D\left(\frac{F\left(t_{0}+\Delta t\right) \sim_{h} F\left(t_{0}\right)}{\Delta t}, F^{\prime}\left(t_{0}\right)\right)=0
$$

and

$$
\lim _{\Delta t \rightarrow 0^{+}} D\left(\frac{F\left(t_{0}\right) \sim_{h} F\left(t_{0}-\Delta t\right)}{\Delta t}, F^{\prime}\left(t_{0}\right)\right)=0
$$

the fuzzy set $F^{\prime}\left(t_{0}\right)$ is called the Hukuhara derivative of $F$ at $t_{0}$.

Recall that $U \sim_{h} V=W \epsilon \mathbb{E}$ are defined on level sets, where $[U]^{\alpha} \sim_{h}[V]^{\alpha}=[W]^{\alpha}$ for all $0 \leq \alpha \leq 1$. By consideration of definition of the metric $D$, all the level set mappings $[F(.)]^{\alpha}$ are Hukuhara differentiable at $t_{0}$ by Hukuhara derivatives $\left[F^{\prime}\left(t_{0}\right)\right]^{\alpha}$ for each $0 \leq \alpha \leq 1$, when $F: T \rightarrow \mathbb{E}$ is Hukuhara differentiable at $t_{0}$ by Hukuhara derivative $F^{\prime}\left(t_{0}\right)$.

Definition 2.5. The fuzzy integral

$$
\int_{a}^{b} y(t) d t, 0 \leq a \leq b \leq 1
$$

is defined by

$$
\left[\int_{a}^{b} y(t) d t\right]_{\alpha}=\left[\int_{a}^{b} \underline{y}^{\alpha}(t) d t, \int_{a}^{b} \bar{y}^{\alpha}(t) d t\right],
$$

provided that the Lebesque integrals on the right exist.

Remark 2.1. If $F: T \rightarrow \mathbb{E}$ is Hukuhara differentiable and its Hukukara derivative $F^{\prime}$ is integrable over $[0,1]$, then

$$
F(t)=F\left(t_{0}\right)+\int_{t_{0}}^{t} F^{\prime}(s) d s
$$

for all values of $t_{0}, t$ where $0 \leq t_{0} \leq t \leq 1$.

Definition 2.6. A mapping $y: I \rightarrow \mathbb{E}$ is called a fuzzy process. We denote

$$
[y(t)]_{\alpha}=\left[\underline{y}^{\alpha}(t), \bar{y}^{\alpha}(t)\right], t \epsilon I, \alpha \epsilon(0,1] .
$$

The Seikkala derivative $y^{\prime}(t)$ of a fuzzy process $y$ is defined by

$$
\left[y^{\prime}(t)\right]_{\alpha}=\left[\left(\underline{y}^{\alpha}\right)^{\prime}(t),\left(\bar{y}^{\alpha}\right)^{\prime}(t)\right], \alpha \epsilon(0,1],
$$

provided the equation defines a fuzzy number $y^{\prime}(t) \epsilon \mathbb{E}$.

Remark 2.2. If $y: I \rightarrow \mathbb{E}$ is Seikkala differentiable and its Seikkala derivative $y^{\prime}$ is integrable over $[0,1]$, then

$$
y(t)=y\left(t_{0}\right)+\int_{t_{0}}^{t} y^{\prime}(s) d s
$$

for all values of $t_{0}, t$ where $t_{0}, t \epsilon I$. 
2.1. A fuzzy Cauchy problem. Consider the first-order fuzzy differential equation $y^{\prime}=f(t, y)$, where $y$ is a fuzzy function of $t, f(t, y)$ is a fuzzy function of crisp variable $t$ and fuzzy variable $y$, and $y^{\prime}$ is Hukuhara or Seikkala fuzzy derivative of $y$. If an initial value $y\left(t_{0}\right)=y_{0}$ is given, a fuzzy cauchy problem of first-order will be obtained as follows:

$$
y^{\prime}(t)=f(t, y(t)), t_{0} \leq t \leq T, y\left(t_{0}\right)=y_{0} .
$$

Sufficient conditions for the existence of a unique solution to Eq. (2.7) are:

(i) Continuity of $f$

(ii) Lipschitz condition

$D(f(t, x), f(t, y)) \leq L D(x, y), L>0$.

By Theorem 5.2 in [35] we may replace Eq. (2.7) by equivalent system

$$
\begin{aligned}
y^{\prime}(t) & =f(t, y)=F(t, \underline{y}, \bar{y}), \underline{y}\left(t_{0}\right)=\underline{y}_{0}, \\
\bar{y}^{\prime}(t) & =\overline{\bar{f}}(t, y)=G(t, \underline{y}, \bar{y}), \bar{y}\left(t_{0}\right)=\bar{y}_{0},
\end{aligned}
$$

which possesses a unique solution $(\underline{y}, \bar{y}]$ which is a fuzzy function, i.e. for each $t$, the pair $[\underline{y}(t ; \alpha), \bar{y}(t ; \alpha)]$ is a fuzzy number.

The parametric form of Eq. (2.8) is given by

$$
\begin{aligned}
y^{\prime}(t ; \alpha) & =F(t, \underline{y}(t ; \alpha), \bar{y}(t ; \alpha)), \underline{y}\left(t_{0} ; \alpha\right)=\underline{y}_{0}^{\alpha}, \\
\bar{y}^{\prime}(t ; \alpha) & =G(t, \underline{y}(t ; \alpha), \bar{y}(t ; \alpha)), \bar{y}\left(t_{0} ; \alpha\right)=\bar{y}_{0}^{\alpha},
\end{aligned}
$$

for $0 \leq \alpha \leq 1$. In some cases the system given by Eq. (2.9) can be solved analytically [29]. In most cases, however analytically solutions may not be found and a numerical approach must be considered. We know for every prefixed $\alpha$, Eq. (2.9) represents an ordinary Cauchy problem for which any convergence classical numerical procedure can be applied. Some numerical methods such as the fuzzy Euler, Adams-Bashforth, Adams-Moulton, Nyström and predictor-corrector in FDE presented in [28],[29],[30],[31].

2.2. Interpolation of fuzzy number. Suppose that at various time instant $t$ information $f(t)$ is presented as fuzzy set. The aim is to approximate the function $f(t)$, for all $t$ in the domain of $f$. Let $t_{0}<t_{1}<\ldots<t_{n}$ be $n+1$ distinct points in $\mathbb{R}$ and let $\widetilde{u}_{0}, \widetilde{u}_{1}, \ldots, \widetilde{u}_{n}$ be $n+1$ fuzzy sets in $\mathbb{E}$. A fuzzy polynomial interpolation of the data is a fuzzy-value continuous function $f: \mathbb{R} \rightarrow \mathbb{E}$ satisfying:

(i) $f\left(t_{i}\right)=\widetilde{u}_{i}, i=0,1, \ldots, n$,

(ii) If the data is crisp, then the interpolation $f$ is a crisp polynomial.

A function $f$ which fulfilling these condition may be constructed as follows.

Let $C_{\alpha}^{i}=\left[\widetilde{u}_{i}\right]^{\alpha}$ for any $\alpha \epsilon[0,1], i=0,1, \ldots, n$. For each $x=\left(x_{0}, x_{1}, \ldots, x_{n}\right) \epsilon \mathbb{R}^{n+1}$, the unique polynomial of degree $\leq n$ denoted by $P_{x}$ such that

$$
P_{x}\left(t_{i}\right)=x_{i}, i=0,1, \ldots, n, \quad P_{x}(t)=\sum_{i=0}^{n} x_{i}\left(\prod_{i \neq j} \frac{t-t_{j}}{t_{i}-t_{j}}\right) .
$$

Finally, for each $t \in \mathbb{R}$ and $\xi \in \mathbb{R}$ is defined by $f(t) \in \mathbb{E}$ by

$$
(f(t))(\xi)=\sup \left\{\alpha \epsilon[0,1]: \exists x \epsilon C_{\alpha}^{0} \times \ldots \times C_{\alpha}^{n} \text { such that } P_{x}(t)=\xi\right\} .
$$

The interpolation polynomial can be written level set wise as

$$
[f(t)]^{\alpha}=\left\{y \in \mathbb{R}: y=P_{x}(t), x \epsilon\left[\widetilde{u}_{i}\right]^{\alpha}, i=1,2, \ldots, n\right\},
$$


for $0 \leq \alpha \leq 1$. When the data $\widetilde{u_{i}}$, presents as triangular fuzzy numbers, values of the interpolation polynomial are also triangular fuzzy numbers. Then $f(t)$ has a particular simple form that is well suited to computation. The next result is proved in [36].

Theorem 2.2. Let $\left(t_{i}, \widetilde{u_{i}}\right), i=0,1, \ldots, n$ be the observed data and suppose that each of the $\widetilde{u_{i}}=\left(u_{i}^{l}, u_{i}^{c}, u_{i}^{r}\right)$ is an element of $\mathbb{E}$. Then for each $t \epsilon\left[t_{0}, t_{n}\right], \widetilde{f}(t)=$ $\left(f^{l}(t), f^{c}(t), f^{r}(t)\right) \epsilon E$,

$$
\begin{array}{r}
f^{l}(t)=\sum_{l_{i}(t) \geq 0} l_{i}(t) u_{i}^{l}+\sum_{l_{i}(t)<0} l_{i}(t) u_{i}^{r}, \\
f^{c}(t)=\sum_{i=0}^{n} l_{i}(t) u_{i}^{c}, \\
f^{r}(t)=\sum_{l_{i}(t) \geq 0} l_{i}(t) u_{i}^{r}+\sum_{l_{i}(t)<0} l_{i}(t) u_{i}^{l},
\end{array}
$$

such that

$$
l_{i}(t)=\prod_{i \neq j} \frac{t-t_{j}}{t_{i}-t_{j}} .
$$

Proof. See [36].

\section{MiLne's PREDICTOR-CORRECTOR METHOD}

We are going to solve fuzzy initial value problem $y^{\prime}(t)=f(t, y(t))$ by Milne's predictor-corrector method. Let the fuzzy initial values be $\widetilde{y}\left(t_{i-3}\right), \widetilde{y}\left(t_{i-2}\right), \widetilde{y}\left(t_{i-1}\right), \widetilde{y}\left(t_{i}\right)$, i.e. $\widetilde{f}\left(t_{i-3}, y\left(t_{i-3}\right)\right), \widetilde{f}\left(t_{i-2}, y\left(t_{i-2}\right)\right), \widetilde{f}\left(t_{i-1}, y\left(t_{i-1}\right)\right), \widetilde{f}\left(t_{i}, y\left(t_{i}\right)\right)$, which are triangular fuzzy numbers are shown by

$$
\begin{array}{r}
\left\{f^{l}\left(t_{i-3}, y\left(t_{i-3}\right)\right), f^{c}\left(t_{i-3}, y\left(t_{i-3}\right)\right), f^{r}\left(t_{i-3}, y\left(t_{i-3}\right)\right)\right\} \\
\left\{f^{l}\left(t_{i-2}, y\left(t_{i-2}\right)\right), f^{c}\left(t_{i-2}, y\left(t_{i-2}\right)\right), f^{r}\left(t_{i-2}, y\left(t_{i-2}\right)\right)\right\} \\
\left\{f^{l}\left(t_{i-1}, y\left(t_{i-1}\right)\right), f^{c}\left(t_{i-1}, y\left(t_{i-1}\right)\right), f^{r}\left(t_{i-1}, y\left(t_{i-1}\right)\right)\right\} \\
\left\{f^{l}\left(t_{i}, y\left(t_{i}\right)\right), f^{c}\left(t_{i}, y\left(t_{i}\right)\right), f^{r}\left(t_{i}, y\left(t_{i}\right)\right)\right\} .
\end{array}
$$

Firstly, considering the quadratic Lagrange polynomial interpolating $\widetilde{P_{2}}(t)$ at point $t_{i-2}, t_{i-1}, t_{i}$ and integrating over $\left[t_{i-3}, t_{i+1}\right]$, we have

$$
\begin{gathered}
\widetilde{y}\left(t_{i+1}\right)=\widetilde{y}\left(t_{i-3}\right)+\int_{t_{i-3}}^{t_{i+1}} \widetilde{f}(t, y(t)) d t \\
\approx \widetilde{y}\left(t_{i-3}\right)+\int_{t_{i-3}}^{t_{i+1}} \widetilde{P_{2}}(t) d t .
\end{gathered}
$$

By taking $\widetilde{f}\left(t_{i-2}, y\left(t_{i-2}\right)\right), \widetilde{f}\left(t_{i-1}, y\left(t_{i-1}\right)\right), \widetilde{f}\left(t_{i}, y\left(t_{i}\right)\right)$, we get:

$$
f_{x}^{l}(t, y(t))=\sum_{\substack{j=i-2 \\ l_{j}(t) \geq 0}}^{i} l_{j}(t) f^{l}\left(t_{j}, y\left(t_{j}\right)\right)+\sum_{\substack{j=i-2 \\ l_{j}(t)<0}}^{i} l_{j}(t) f^{r}\left(t_{j}, y\left(t_{j}\right)\right)
$$




$$
\begin{gathered}
f_{x}^{c}(t, y(t))=\sum_{j=i-2}^{i} l_{j}(t) f^{c}\left(t_{j}, y\left(t_{j}\right)\right) \\
f_{x}^{r}(t, y(t))=\sum_{\substack{j=i-2 \\
l_{j}(t) \geq 0}}^{i} l_{j}(t) f^{r}\left(t_{j}, y\left(t_{j}\right)\right)+\sum_{\substack{j=i-2 \\
l_{j}(t)<0}}^{i} l_{j}(t) f^{l}\left(t_{j}, y\left(t_{j}\right)\right)
\end{gathered}
$$

for $t_{i-3} \leq t \leq t_{i+1}$ :

$$
\begin{array}{r}
l_{i-2}(t)=\frac{\left(t-t_{i-1}\right)\left(t-t_{i}\right)}{\left(t_{i-2}-t_{i-1}\right)\left(t_{i-2}-t_{i}\right)} \geq 0, \\
l_{i-1}(t)=\frac{\left(t-t_{i-2}\right)\left(t-t_{i}\right)}{\left(t_{i-1}-t_{i-2}\right)\left(t_{i-1}-t_{i}\right)} \leq 0, \\
l_{i}(t)=\frac{\left(t-t_{i-2}\right)\left(t-t_{i-1}\right)}{\left(t_{i}-t_{i-2}\right)\left(t_{i}-t_{i-1}\right)} \geq 0,
\end{array}
$$

therefore the following results will be obtained:

$$
f^{l}(t, y(t))=l_{i-2}(t) f^{l}\left(t_{i-2}, y\left(t_{i-2}\right)\right)+l_{i-1}(t) f^{r}\left(t_{i-1}, y\left(t_{i-1}\right)\right)+l_{i}(t) f^{l}\left(t_{i}, y\left(t_{i}\right)\right)
$$

$$
f^{c}(t, y(t))=l_{i-2}(t) f^{c}\left(t_{i-2}, y\left(t_{i-2}\right)\right)+l_{i-1}(t) f^{c}\left(t_{i-1}, y\left(t_{i-1}\right)\right)+l_{i}(t) f^{c}\left(t_{i}, y\left(t_{i}\right)\right)
$$

$$
f^{r}(t, y(t))=l_{i-2}(t) f^{r}\left(t_{i-2}, y\left(t_{i-2}\right)\right)+l_{i-1}(t) f^{l}\left(t_{i-1}, y\left(t_{i-1}\right)\right)+l_{i}(t) f^{r}\left(t_{i}, y\left(t_{i}\right)\right)
$$

From (2.6) and (3.1) it follows that:

$$
\widetilde{y}^{\alpha}\left(t_{i+1}\right)=\left[\underline{y}^{\alpha}\left(t_{i+1}\right), \bar{y}^{\alpha}\left(t_{i+1}\right)\right]
$$

where

$$
\underline{y}^{\alpha}\left(t_{i+1}\right)=\underline{y}^{\alpha}\left(t_{i-3}\right)+\int_{t_{i-3}}^{t_{i+1}}\left\{\alpha f^{c}(t, y(t))+(1-\alpha) f^{l}(t, y(t))\right\} d t
$$

and

$$
\bar{y}^{\alpha}\left(t_{i+1}\right)=\bar{y}^{\alpha}\left(t_{i-3}\right)+\int_{t_{i-3}}^{t_{i+1}}\left\{\alpha f^{c}(t, y(t))+(1-\alpha) f^{r}(t, y(t))\right\} d t
$$

If (3.2) and (3.3) are situated in (3.5) and (3.3), (3.4) in (3.6):

$$
\begin{aligned}
& \underline{y}^{\alpha}\left(t_{i+1}\right)=\underline{y}^{\alpha}\left(t_{i-3}\right)+\int_{t_{i-3}}^{t_{i+1}}\left\{\alpha\left[l_{i-2}(t) f^{c}\left(t_{i-2}, y\left(t_{i-2}\right)\right)+l_{i-1}(t) f^{c}\left(t_{i-1}, y\left(t_{i-1}\right)\right)+l_{i}(t) f^{c}\left(t_{i}, y\left(t_{i}\right)\right)\right]\right. \\
& \left.+(1-\alpha)\left[l_{i-2}(t) f^{l}\left(t_{i-2}, y\left(t_{i-2}\right)\right)+l_{i-1}(t) f^{r}\left(t_{i-1}, y\left(t_{i-1}\right)\right)+l_{i}(t) f^{l}\left(t_{i}, y\left(t_{i}\right)\right)\right]\right\} d t \\
& \text { and } \\
& \bar{y}^{\alpha}\left(t_{i+1}\right)=\bar{y}^{\alpha}\left(t_{i-3}\right)+\int_{t_{i-3}}^{t_{i+1}}\left\{\alpha\left[l_{i-2}(t) f^{c}\left(t_{i-2}, y\left(t_{i-2}\right)\right)+l_{i-1}(t) f^{c}\left(t_{i-1}, y\left(t_{i-1}\right)\right)+l_{i}(t) f^{c}\left(t_{i}, y\left(t_{i}\right)\right)\right]\right. \\
& \left.+(1-\alpha)\left[l_{i-2}(t) f^{r}\left(t_{i-2}, y\left(t_{i-2}\right)\right)+l_{i-1}(t) f^{l}\left(t_{i-1}, y\left(t_{i-1}\right)\right)+l_{i}(t) f^{r}\left(t_{i}, y\left(t_{i}\right)\right)\right]\right\} d t \\
& \text { The following results will be obtained by integration: }
\end{aligned}
$$$$
\underline{y}^{\alpha}\left(t_{i+1}\right)=\underline{y}^{\alpha}\left(t_{i-3}\right)+\frac{8 h}{3}\left[\alpha f^{c}\left(t_{i-2}, y\left(t_{i-2}\right)\right)+(1-\alpha) f^{l}\left(t_{i-2}, y\left(t_{i-2}\right)\right)\right]
$$$$
-\frac{4 h}{3}\left[\alpha f^{c}\left(t_{i-1}, y\left(t_{i-1}\right)\right)+(1-\alpha) f^{r}\left(t_{i-1}, y\left(t_{i-1}\right)\right)\right]+\frac{8 h}{3}\left[\alpha f^{c}\left(t_{i}, y\left(t_{i}\right)\right)+(1-\alpha) f^{l}\left(t_{i}, y\left(t_{i}\right)\right)\right]
$$
and 
$\bar{y}^{\alpha}\left(t_{i+1}\right)=\bar{y}^{\alpha}\left(t_{i-3}\right)+\frac{8 h}{3}\left[\alpha f^{c}\left(t_{i-2}, y\left(t_{i-2}\right)\right)+(1-\alpha) f^{r}\left(t_{i-2}, y\left(t_{i-2}\right)\right)\right]$

$-\frac{4 h}{3}\left[\alpha f^{c}\left(t_{i-1}, y\left(t_{i-1}\right)\right)+(1-\alpha) f^{l}\left(t_{i-1}, y\left(t_{i-1}\right)\right)\right]+\frac{8 h}{3}\left[\alpha f^{c}\left(t_{i}, y\left(t_{i}\right)\right)+(1-\alpha) f^{r}\left(t_{i}, y\left(t_{i}\right)\right)\right]$

Thus

$$
\underline{y}^{\alpha}\left(t_{i+1}\right)=\underline{y}^{\alpha}\left(t_{i-3}\right)+\frac{4 h}{3}\left[2 \underline{f}^{\alpha}\left(t_{i-2}, y\left(t_{i-2}\right)\right)-\bar{f}^{\alpha}\left(t_{i-1}, y\left(t_{i-1}\right)\right)+2 \underline{f}^{\alpha}\left(t_{i}, y\left(t_{i}\right)\right)\right]
$$

$$
\bar{y}^{\alpha}\left(t_{i+1}\right)=\bar{y}^{\alpha}\left(t_{i-3}\right)+\frac{4 h}{3}\left[2 \bar{f}^{\alpha}\left(t_{i-2}, y\left(t_{i-2}\right)\right)-\underline{f}^{\alpha}\left(t_{i-1}, y\left(t_{i-1}\right)\right)+2 \bar{f}^{\alpha}\left(t_{i}, y\left(t_{i}\right)\right)\right]
$$

Therefore, in Milne's method as a predictor formula is obtained as follows:

$$
\begin{aligned}
y^{\alpha}\left(t_{i+1}\right) & =y^{\alpha}\left(t_{i-3}\right)+\frac{4 h}{3}\left[2 f^{\alpha}\left(t_{i-2}, y\left(t_{i-2}\right)\right)-\bar{f}^{\alpha}\left(t_{i-1}, y\left(t_{i-1}\right)\right)+2 f^{\alpha}\left(t_{i}, y\left(t_{i}\right)\right)\right] \\
\bar{y}^{\alpha}\left(t_{i+1}\right) & =\overline{\bar{y}}^{\alpha}\left(t_{i-3}\right)+\frac{4 h}{3}\left[2 \overline{\bar{f}}^{\alpha}\left(t_{i-2}, y\left(t_{i-2}\right)\right)-f^{\alpha}\left(t_{i-1}, y\left(t_{i-1}\right)\right)+2 \overline{\bar{f}}^{\alpha}\left(t_{i}, y\left(t_{i}\right)\right)\right] \\
y^{\alpha}\left(t_{i-3}\right) & =\alpha_{0}, y^{\alpha}\left(t_{i-2}\right)=\alpha_{1}, y^{\alpha}\left(t_{i-1}\right)=\alpha_{2}, y^{\alpha}\left(t_{i}\right)=\alpha_{3}, \\
\overline{\bar{y}}^{\alpha}\left(t_{i-3}\right) & =\alpha_{4}, \overline{\bar{y}}^{\alpha}\left(t_{i-2}\right)=\alpha_{5}, \overline{\bar{y}}^{\alpha}\left(t_{i-1}\right)=\alpha_{6}, \overline{\bar{y}}^{\alpha}\left(t_{i}\right)=\alpha_{7} .
\end{aligned}
$$

Secondly, considering the polynomial interpolating $\widetilde{P_{2}}(t)$, which fits at points $t_{i-1}, t_{i}, t_{i+1}$ and integrating over $\left[t_{i-1}, t_{i+1}\right]$, we have

$$
\begin{aligned}
& \widetilde{y}\left(t_{i+1}\right)=\widetilde{y}\left(t_{i-1}\right)+\int_{t_{i-1}}^{t_{i+1}} \widetilde{f}(t, y(t)) d t \\
& \approx \int_{t_{i-1}}^{t_{i+1}} \widetilde{y}\left(t_{i-1}\right)+\widetilde{P_{2}}(t) d t
\end{aligned}
$$

By taking $\widetilde{f}\left(t_{i-1}, y\left(t_{i-1}\right)\right), \quad \widetilde{f}\left(t_{i}, y\left(t_{i}\right)\right), \widetilde{f}\left(t_{i+1}, y\left(t_{i+1}\right)\right)$, we get:

$$
\begin{aligned}
f^{l}(t, y(t))= & \sum_{\substack{j=i-1 \\
l_{j}(t) \geq 0}}^{i+1} l_{j}(t) f^{l}\left(t_{j}, y\left(t_{j}\right)\right) \\
f^{c}(t, y(t))= & \sum_{j=i-1}^{i+1} l_{j}(t) f^{c}\left(t_{j}, y\left(t_{j}\right)\right) \\
f^{r}(t, y(t))= & \sum_{j=i-1}^{i+1} l_{j}(t) f^{r}\left(t_{j}, y\left(t_{j}\right)\right) \\
& l_{j}(t) \geq 0
\end{aligned}
$$

for $t_{i-1} \leq t \leq t_{i+1}$ :

$$
\begin{array}{r}
l_{i-1}(t)=\frac{\left(t-t_{i}\right)\left(t-t_{i+1}\right)}{\left(t_{i-1}-t_{i}\right)\left(t_{i-1}-t_{i+1}\right)} \geq 0, \\
l_{i}(t)=\frac{\left(t-t_{i-1}\right)\left(t-t_{i+1}\right)}{\left(t_{i}-t_{i-1}\right)\left(t_{i}-t_{i+1}\right)} \geq 0, \\
l_{i+1}(t)=\frac{\left(t-t_{i-1}\right)\left(t-t_{i}\right)}{\left(t_{i+1}-t_{i-1}\right)\left(t_{i+1}-t_{i}\right)} \geq 0
\end{array}
$$

therefore the following results will be obtained:

(3.11)

$$
f^{l}(t, y(t))=l_{i-1}(t) f^{l}\left(t_{i-1}, y\left(t_{i-1}\right)\right)+l_{i}(t) f^{l}\left(t_{i}, y\left(t_{i}\right)\right)+l_{i+1}(t) f^{l}\left(t_{i+1}, y\left(t_{i+1}\right)\right)
$$


$(3.12)$

$$
f^{c}(t, y(t))=l_{i-1}(t) f^{c}\left(t_{i-1}, y\left(t_{i-1}\right)\right)+l_{i}(t) f^{c}\left(t_{i}, y\left(t_{i}\right)\right)+l_{i+1}(t) f^{c}\left(t_{i+1}, y\left(t_{i+1}\right)\right)
$$

$$
f^{r}(t, y(t))=l_{i-1}(t) f^{r}\left(t_{i-1}, y\left(t_{i-1}\right)\right)+l_{i}(t) f^{r}\left(t_{i}, y\left(t_{i}\right)\right)+l_{i+1}(t) f^{r}\left(t_{i+1}, y\left(t_{i+1}\right)\right)
$$

From (2.6) and (3.10) it follows that:

$$
\widetilde{y}^{\alpha}\left(t_{i+1}\right)=\left[\underline{y}^{\alpha}\left(t_{i+1}\right), \bar{y}^{\alpha}\left(t_{i+1}\right)\right],
$$

where

$$
\underline{y}^{\alpha}\left(t_{i+1}\right)=\underline{y}^{\alpha}\left(t_{i-1}\right)+\int_{t_{i-1}}^{t_{i+1}}\left\{\alpha f^{c}(t, y(t))+(1-\alpha) f^{l}(t, y(t))\right\} d t
$$

and

$$
\bar{y}^{\alpha}\left(t_{i+1}\right)=\bar{y}^{\alpha}\left(t_{i-1}\right)+\int_{t_{i-1}}^{t_{i+1}}\left\{\alpha f^{c}(t, y(t))+(1-\alpha) f^{r}(t, y(t))\right\} d t
$$

If (3.11) and (3.12) are situated in (3.14) and (3.12), (3.13) in (3.15):

$$
\begin{aligned}
& \underline{y}^{\alpha}\left(t_{i+1}\right)=\underline{y}^{\alpha}\left(t_{i-1}\right)+\int_{t_{i-1}}^{t_{i+1}}\left\{\alpha\left[l_{i-1}(t) f^{c}\left(t_{i-1}, y\left(t_{i-1}\right)\right)+l_{i}(t) f^{c}\left(t_{i}, y\left(t_{i}\right)\right)+l_{i+1}(t) f^{c}\left(t_{i+1}, y\left(t_{i+1}\right)\right)\right]\right. \\
& \left.+(1-\alpha)\left[l_{i-1}(t) f^{l}\left(t_{i-1}, y\left(t_{i-1}\right)\right)+l_{i}(t) f^{l}\left(t_{i}, y\left(t_{i}\right)\right)+l_{i+1}(t) f^{l}\left(t_{i+1}, y\left(t_{i+1}\right)\right)\right]\right\} d t
\end{aligned}
$$

and

$$
\begin{aligned}
& \bar{y}^{\alpha}\left(t_{i+1}\right)=\bar{y}^{\alpha}\left(t_{i-1}\right)+\int_{t_{i-1}}^{t_{i+1}}\left\{\alpha\left[l_{i-1}(t) f^{c}\left(t_{i-1}, y\left(t_{i-1}\right)\right)+l_{i}(t) f^{c}\left(t_{i}, y\left(t_{i}\right)\right)+l_{i+1}(t) f^{c}\left(t_{i+1}, y\left(t_{i+1}\right)\right)\right]\right. \\
& \left.+(1-\alpha)\left[l_{i-1}(t) f^{r}\left(t_{i-1}, y\left(t_{i-1}\right)\right)+l_{i}(t) f^{r}\left(t_{i}, y\left(t_{i}\right)\right)+l_{i+1}(t) f^{r}\left(t_{i+1}, y\left(t_{i+1}\right)\right)\right]\right\} d t .
\end{aligned}
$$

The following results will be obtained by integration:

$$
\begin{aligned}
& \underline{y}^{\alpha}\left(t_{i+1}\right)=\underline{y}^{\alpha}\left(t_{i-1}\right)+\frac{h}{3}\left[\alpha f^{c}\left(t_{i-1}, y\left(t_{i-1}\right)\right)+(1-\alpha) f^{l}\left(t_{i-1}, y\left(t_{i-1}\right)\right)\right] \\
& +\frac{4 h}{3}\left[\alpha f^{c}\left(t_{i}, y\left(t_{i}\right)\right)+(1-\alpha) f^{l}\left(t_{i}, y\left(t_{i}\right)\right)\right]+\frac{h}{3}\left[\alpha f^{c}\left(t_{i+1}, y\left(t_{i+1}\right)\right)+(1-\alpha) f^{l}\left(t_{i+1}, y\left(t_{i+1}\right)\right)\right]
\end{aligned}
$$

and

$\bar{y}^{\alpha}\left(t_{i+1}\right)=\bar{y}^{\alpha}\left(t_{i-1}\right)+\frac{h}{3}\left[\alpha f^{c}\left(t_{i-1}, y\left(t_{i-1}\right)\right)+(1-\alpha) f^{r}\left(t_{i-1}, y\left(t_{i-1}\right)\right)\right]$

$+\frac{4 h}{3}\left[\alpha f^{c}\left(t_{i}, y\left(t_{i}\right)\right)+(1-\alpha) f^{r}\left(t_{i}, y\left(t_{i}\right)\right)\right]+\frac{h}{3}\left[\alpha f^{c}\left(t_{i+1}, y\left(t_{i+1}\right)\right)+(1-\alpha) f^{r}\left(t_{i+1}, y\left(t_{i+1}\right)\right)\right]$

Thus

$$
\underline{y}^{\alpha}\left(t_{i+1}\right)=\underline{y}^{\alpha}\left(t_{i-1}\right)+\frac{h}{3}\left[\underline{f}^{\alpha}\left(t_{i-1}, y\left(t_{i-1}\right)\right)+4 \underline{f}^{\alpha}\left(t_{i}, y\left(t_{i}\right)\right)+\underline{f}^{\alpha}\left(t_{i+1}, y\left(t_{i+1}\right)\right)\right]
$$

$$
\bar{y}^{\alpha}\left(t_{i+1}\right)=\bar{y}^{\alpha}\left(t_{i-1}\right)+\frac{h}{3}\left[\bar{f}^{\alpha}\left(t_{i-1}, y\left(t_{i-1}\right)\right)+4 \bar{f}^{\alpha}\left(t_{i}, y\left(t_{i}\right)\right)+\bar{f}^{\alpha}\left(t_{i+1}, y\left(t_{i+1}\right)\right)\right]
$$

Therefore, in Milne's method as a corrector formula is obtained as follows:

$$
\begin{aligned}
y^{\alpha}\left(t_{i+1}\right) & =y^{\alpha}\left(t_{i-1}\right)+\frac{h}{3}\left[f^{\alpha}\left(t_{i-1}, y\left(t_{i-1}\right)\right)+4 f^{\alpha}\left(t_{i}, y\left(t_{i}\right)\right)+f^{\alpha}\left(t_{i+1}, y\left(t_{i+1}\right)\right)\right] \\
\bar{y}^{\alpha}\left(t_{i+1}\right) & =\overline{\bar{y}}^{\alpha}\left(t_{i-1}\right)+\frac{h}{3}\left[\overline{\bar{f}}^{\alpha}\left(t_{i-1}, y\left(t_{i-1}\right)\right)+4 \overline{\bar{f}}^{\alpha}\left(t_{i}, y\left(t_{i}\right)\right)+\overline{\bar{f}}^{\alpha}\left(t_{i+1}, y\left(t_{i+1}\right)\right)\right] \\
y^{\alpha}\left(t_{i-1}\right) & =\alpha_{0}, y^{\alpha}\left(t_{i}\right)=\alpha_{1}, y^{\alpha}\left(t_{i+1}\right)=\alpha_{2}, \\
\overline{\bar{y}}^{\alpha}\left(t_{i-1}\right) & =\alpha_{3}, \overline{\bar{y}}^{\alpha}\left(t_{i}\right)=\alpha_{4}, \overline{\bar{y}}^{\alpha}\left(t_{i+1}\right)=\alpha_{5} .
\end{aligned}
$$

The following algorithm is based on Adams-Bashforth method of fourth order as a predictor and also Milne-Simpson method of fourth order as a corrector formula.

To approximate the solution of following fuzzy initial value problem

$$
\begin{aligned}
& y^{\prime}(t)=f(t, y(t)), t_{0} \leq t \leq T \\
& y^{\alpha}\left(t_{0}\right)=\alpha_{0}, y^{\alpha}\left(t_{1}\right)=\alpha_{1}, y^{\alpha}\left(t_{2}\right)=\alpha_{2}, y^{\alpha}\left(t_{3}\right)=\alpha_{3} \\
& \overline{\bar{y}}^{\alpha}\left(t_{0}\right)=\alpha_{4}, \overline{\bar{y}}^{\alpha}\left(t_{1}\right)=\alpha_{5}, \overline{\bar{y}}^{\alpha}\left(t_{2}\right)=\alpha_{6}, \overline{\bar{y}}^{\alpha}\left(t_{3}\right)=\alpha_{7}
\end{aligned}
$$


positive integer $N$ is chosen.

Step 1 . Let $h=\frac{T-t_{0}}{N}$.

Compute starting values using fourth order Runge-Kutta method

$$
\begin{gathered}
\underline{w}^{\alpha}\left(t_{0}\right)=\alpha_{0}, \underline{w}^{\alpha}\left(t_{1}\right)=\alpha_{1}, \\
\underline{w}^{\alpha}\left(t_{2}\right)=\alpha_{2}, \underline{w}^{\alpha}\left(t_{3}\right)=\alpha_{3} \\
\bar{w}^{\alpha}\left(t_{0}\right)=\alpha_{4}, \bar{w}^{\alpha}\left(t_{1}\right)=\alpha_{5}, \\
\bar{w}^{\alpha}\left(t_{2}\right)=\alpha_{6}, \bar{w}^{\alpha}\left(t_{3}\right)=\alpha_{7}
\end{gathered}
$$

Step 2. Let $i=1$.

Step 3. Let

$\underline{w}^{(0) \alpha}\left(t_{i+1}\right)=\underline{w}^{\alpha}\left(t_{i-3}\right)+\frac{4 h}{3}\left[2 \underline{f}^{\alpha}\left(t_{i-2}, w\left(t_{i-2}\right)\right)-\bar{f}^{\alpha}\left(t_{i-1}, w\left(t_{i-1}\right)\right)+2 \underline{f}^{\alpha}\left(t_{i}, w\left(t_{i}\right)\right)\right]$,

$\bar{w}^{(0) \alpha}\left(t_{i+1}\right)=\bar{w}^{\alpha}\left(t_{i-3}\right)+\frac{4 h}{3}\left[2 \overline{\bar{f}}^{\alpha}\left(t_{i-2}, w\left(t_{i-2}\right)\right)-\underline{f}^{\alpha}\left(t_{i-1}, w\left(t_{i-1}\right)\right)+2 \overline{\bar{f}}^{\alpha}\left(t_{i}, w\left(t_{i}\right)\right)\right]$,

Step 4. Let

$t_{i+2}=t_{0}+(i+2) h$

Step 5. Let

$\underline{w}^{\alpha}\left(t_{i+1}\right)=\underline{w}^{\alpha}\left(t_{i-1}\right)+\frac{h}{3}\left[\underline{f}^{\alpha}\left(t_{i-1}, w\left(t_{i-1}\right)\right)+4 \underline{f}^{\alpha}\left(t_{i}, w\left(t_{i}\right)\right)+\underline{f}^{\alpha}\left(t_{i+1}, w^{(0)}\left(t_{i+1}\right)\right)\right]$,

$\bar{w}^{\alpha}\left(t_{i+1}\right)=\bar{w}^{\alpha}\left(t_{i-1}\right)+\frac{h}{3}\left[\bar{f}^{\alpha}\left(t_{i-1}, w\left(t_{i-1}\right)\right)+4 \overline{\bar{f}}^{\alpha}\left(t_{i}, w\left(t_{i}\right)\right)+\overline{\bar{f}}^{\alpha}\left(t_{i+1}, w^{(0)}\left(t_{i+1}\right)\right)\right]$,

Step 6. $i=i+1$.

Step 7. if $i \leq N-1$ go to Step 3 .

Step 8. Algorithm will be completed on $\left(\underline{w}^{\alpha}(T), \bar{w}^{\alpha}(T)\right)$ approximates real value of $\left(\underline{Y}^{\alpha}(T), \bar{Y}^{\alpha}(T)\right)$.

\section{Convergence And Stability}

To integrate the system given in Eq. (2.9) from to a prefixed $T>t_{0}$, the interval $\left[t_{0}, T\right]$ will be replaced by a set of discrete equally spaced grid points $t_{0}<t_{1}<$ $\ldots<t_{N}=T$ at which the exact solution $(\underline{Y}(t ; \alpha), \bar{Y}(t ; \alpha))$ is approximated by some $(y(t ; \alpha), \bar{y}(t ; \alpha))$. The exact and approximate solutions at $t_{n}, 0 \leq n \leq N$ are denoted by $Y_{n}(\alpha)=\left[\underline{Y}_{n}(\alpha), \bar{Y}_{n}(\alpha)\right]$, and $y_{n}(\alpha)=\left[\underline{y}_{n}(\alpha), \bar{y}_{n}(\alpha)\right]$,respectively. The grid points at which the solution is calculated are $t_{n}=t_{0}+n h, h=\frac{T-t_{0}}{N}, 0 \leq n \leq$ $N$.

From Eq. (3.18), the polygon curves

$$
\begin{aligned}
& \underline{y}(t ; h ; \alpha)=\left\{\left|t_{0}, \underline{y_{0}(\alpha)}\right|,\left|t_{1}, \underline{y_{1}(\alpha)}\right|, \ldots,\left|t_{N}, \underline{y_{N}(\alpha)}\right|\right\}, \\
& \bar{y}(t ; h ; \alpha)=\left\{\left|t_{0}, \overline{y_{0}(\alpha)}\right|,\left|t_{1}, \overline{y_{1}(\alpha)}\right|, \ldots,\left|t_{N}, \overline{y_{N}(\alpha)}\right|\right\},
\end{aligned}
$$

are the Milne's predictor-corrector method approximates to $\underline{Y}(t, \alpha)$ and $\bar{Y}(t, \alpha)$, respectively, over the interval $t_{0} \leq t \leq t_{N}$. The following lemma will be applied to show convergence of these approximates, i.e.

$$
\lim _{h \rightarrow 0} \underline{y}(t ; h ; \alpha)=\underline{Y}(t ; \alpha), \lim _{h \rightarrow 0} \bar{y}(t ; h ; \alpha)=\bar{Y}(t ; \alpha) .
$$

Lemma 4.1. [28] Let a sequence of numbers $\left\{W_{n}\right\}_{n=0}^{N}$ satisfy:

$$
\left|W_{n+1}\right| \leq A\left|W_{n}\right|+B\left|W_{n-1}\right|+C, 0 \leq n \leq N-1
$$

for some given positive constants $A, B$ and $C$. Then

$\left|W_{n}\right| \leq\left(A^{n-1}+\beta_{1} A^{n-3} B+\beta_{2} A^{n-5} B^{2}+\ldots+\beta_{s} B^{\left[\frac{n}{2}\right]}\right)\left|W_{1}\right|$

$+\left(A^{n-2} B+\gamma_{1} A^{n-4} B^{2}+\ldots+\gamma_{t} A B^{\left[\frac{n}{2}\right]}\right)\left|W_{0}\right|+\left(A^{n-2}+A^{n-3}+\ldots+1\right) C$ 
$+\left(\delta_{1} A^{n-4}+\delta_{2} A^{n-5}+\ldots+\delta_{m} A+1\right) B C+\left(\xi_{1} A^{n-6}+\xi_{2} A^{n-7}+\ldots+\xi_{r} A+1\right) B^{2} C$ $+\left(\lambda_{1} A^{n-8}+\lambda_{2} A^{n-9}+\ldots+\lambda_{p} A+1\right) B^{3} C+\ldots, n$ odd and

$\left|W_{n}\right| \leq\left(A^{n-1}+\beta_{1} A^{n-3} B+\beta_{2} A^{n-5} B^{2}+\ldots+\beta_{s} A B^{\left[\frac{n}{2}\right]-1}\right)\left|W_{1}\right|$

$+\left(A^{n-2} B+\gamma_{1} A^{n-4} B^{2}+\ldots+\gamma_{t} B^{\left[\frac{n}{2}\right]}\right)\left|W_{0}\right|+\left(A^{n-2}+A^{n-3}+\ldots+1\right) C$

$+\left(\delta_{1} A^{n-4}+\delta_{2} A^{n-5}+\ldots+\delta_{m} A+1\right) B C+\left(\xi_{1} A^{n-6}+\xi_{2} A^{n-7}+\ldots+\xi_{r} A+1\right) B^{2} C$

$+\left(\lambda_{1} A^{n-8}+\lambda_{2} A^{n-9}+\ldots+\lambda_{p} A+1\right) B^{3} C+\ldots, n$ even

where $\beta_{s}, \gamma_{t}, \delta_{m}, \xi_{r}, \lambda_{p}$ are constants for all $s, t, m, r$ and $p$.

The proof, by using mathematical induction is straightforward.

Theorem 4.1. For arbitrary fixed $\alpha: 0 \leq \alpha \leq 1$, the Milne-Simpson of fourth order approximates of Eq.(3.9) converge to the exact solutions $\underline{Y}(t ; \alpha), \bar{Y}(t ; \alpha)$, uniformly in $t$, for $\underline{Y}, \bar{Y} \epsilon C^{5}\left[t_{0}, T\right]$.

Proof. It is sufficient to show

$$
\lim _{h \rightarrow 0} \underline{y}_{n}(\alpha)=\underline{Y}(T ; \alpha), \lim _{h \rightarrow 0} \bar{y}_{n}(\alpha)=\bar{Y}(T ; \alpha)
$$

By using Taylor's theorem, we get

$\underline{Y}_{n+1}(\alpha)=\underline{Y}_{n-1}(\alpha)+\frac{h}{3} f\left(t_{n-1}, \underline{Y}_{n-1}(\alpha)\right)+\frac{4 h}{3} f\left(t_{n}, \underline{Y}_{n}(\alpha)\right)+\frac{h}{3} f\left(t_{n+1}, \underline{Y}_{n+1}(\alpha)\right)-$ $\frac{1}{90} h^{5} \underline{Y}^{(V)}\left(\xi_{n}\right)$,

$\bar{Y}_{n+1}(\alpha)=\bar{Y}_{n-1}(\alpha)+\frac{h}{3} f\left(t_{n-1}, \bar{Y}_{n-1}(\alpha)\right)+\frac{4 h}{3} f\left(t_{n}, \bar{Y}_{n}(\alpha)\right)+\frac{h}{3} f\left(t_{n+1}, \bar{Y}_{n+1}(\alpha)\right)-$ $\frac{1}{90} h^{5} \bar{Y}^{(V)}\left(\xi_{n}\right)$

where $t_{n-3}<\underline{\xi}_{n}, \bar{\xi}_{n}<t_{n+1}$.

Consequently,

$\underline{Y}_{n+1}(\alpha)-\underline{y}_{n+1}(\alpha)=\underline{Y}_{n-1}(\alpha)-\underline{y}_{n-1}(\alpha)+\frac{h}{3} f\left(t_{n-1}, \underline{Y}_{n-1}(\alpha)\right)+\frac{4 h}{3} f\left(t_{n}, \underline{Y}_{n}(\alpha)\right)+$ $\frac{h}{3} f\left(t_{n+1}, \underline{Y}_{n+1}(\alpha)\right)-\frac{1}{90} h^{5} \underline{Y}^{(V)}\left(\xi_{n}\right)$,

$\bar{Y}_{n+1}(\alpha)-\bar{y}_{n+1}(\alpha)=\bar{Y}_{n-1}(\alpha)-\bar{y}_{n-1}(\alpha)+\frac{h}{3} f\left(t_{n-1}, \bar{Y}_{n-1}(\alpha)\right)+\frac{4 h}{3} f\left(t_{n}, \bar{Y}_{n}(\alpha)\right)+$ $\frac{h}{3} f\left(t_{n+1}, \bar{Y}_{n+1}(\alpha)\right)-\frac{1}{90} h^{5} \bar{Y}^{(V)}\left(\xi_{n}\right)$,

Denote $W_{n}=\underline{Y}_{n}(\alpha)-\underline{y}_{n}(\alpha), V_{n}=\bar{Y}_{n}(\alpha)-\bar{y}_{n}(\alpha)$. Then

$$
\begin{array}{r}
\left|W_{n+1}\right| \leq\left(1+\frac{h L_{1}}{3}\right)\left|W_{n-1}\right|+\left(\frac{4 h L_{2}}{3}\right)\left|W_{n}\right|+\left(\frac{h L_{3}}{3}\right)\left|W_{n+1}\right|-\frac{1}{90} h^{5} \underline{M} \\
\left|V_{n+1}\right| \leq\left(1+\frac{h L_{4}}{3}\right)\left|V_{n-1}\right|+\left(\frac{4 h L_{5}}{3}\right)\left|V_{n}\right|+\left(\frac{h L_{6}}{3}\right)\left|V_{n+1}\right|-\frac{1}{90} h^{5} \bar{M}
\end{array}
$$

where

$$
\underline{M}=\max _{t_{0} \leq t \leq T}\left|\underline{Y}^{(V)}(t ; \alpha)\right|
$$

and

$$
\bar{M}=\max _{t_{0} \leq t \leq T}\left|\bar{Y}^{(V)}(t ; \alpha)\right|
$$

and is put

$$
L=\max \left\{L_{1}, L_{2}, L_{3}, L_{4}, L_{5}, L_{6}\right\}<\frac{3}{h}
$$


then

$$
\begin{array}{r}
\left|W_{n+1}\right| \leq\left(1+\frac{2 h L}{3-h L}\right)\left|W_{n-1}\right|+\left(\frac{4 h L}{3-h L}\right)\left|W_{n}\right|+\left(\frac{1}{90-30 h L} h^{5} \underline{M}\right), \\
\left|V_{n+1}\right| \leq\left(1+\frac{2 h L}{3-h L}\right)\left|V_{n-1}\right|+\left(\frac{4 h L}{3-h L}\right)\left|V_{n}\right|+\left(\frac{1}{90-30 h L} h^{5} \bar{M}\right)
\end{array}
$$

are resulted, where $\left|U_{n}\right|=\left|W_{n}\right|+\left|V_{n}\right|$ then by Lemma 4.1 and $w_{0}=v_{0}=w_{1}=$ $v_{1}=0$ :

$$
\begin{aligned}
& \left|U_{n}\right| \leq \frac{\left(\frac{4 h L}{3-h L}\right)^{n-1}-1}{\frac{4 h L}{3-h L}} \times\left(\frac{1}{90-30 h L} h^{5}(\underline{M}+\bar{M})\right)+\left\{\delta_{1}\left(\frac{4 h L}{3-h L}\right)^{n-4}+\delta_{2}\left(\frac{4 h L}{3-h L}\right)^{n-5}+\ldots\right. \\
& \left.+\delta_{m}\left(\frac{4 h L}{3-h L}\right)+1\right\}\left(1+\frac{2 h L}{3-h L}\right)^{2}\left(\frac{1}{90-30 h L} h^{5}(\underline{M}+\bar{M})\right)+\left\{\xi_{1}\left(\frac{4 h L}{3-h L}\right)^{n-6}+\xi_{2}\left(\frac{4 h L}{3-h L}\right)^{n-7}+\ldots\right. \\
& \left.+\xi_{r}\left(\frac{4 h L}{3-h L}\right)+1\right\}\left(1+\frac{2 h L}{3-h L}\right)^{3}\left(\frac{1}{90-30 h L} h^{5}(\underline{M}+\bar{M})\right)+\left\{\lambda_{1}\left(\frac{4 h L}{3-h L}\right)^{n-8}+\lambda_{2}\left(\frac{4 h L}{3-h L}\right)^{n-9}+\ldots\right. \\
& \left.+\lambda_{p}\left(\frac{4 h L}{3-h L}\right)+1\right\}\left(1+\frac{2 h L}{3-h L}\right)^{4}\left(\frac{1}{90-30 h L} h^{5}(\underline{M}+\bar{M})\right)+\ldots
\end{aligned}
$$

are obtained. If $h \rightarrow 0$ then $W_{n} \rightarrow 0, V_{n} \rightarrow 0$ which concludes the proof.

Remark 4.1. Above theorem results that convergence order is $O\left(h^{4}\right)$.

Theorem 4.2. For arbitrary fixed $\alpha: 0 \leq \alpha \leq 1$, the Adams-Bashforth of fourth order approximates of Eq.(3.9) converge to the exact solution $\underline{Y}(t ; \alpha), \bar{Y}(t ; \alpha)$, for $\underline{Y}, \bar{Y} \epsilon C^{5}\left[t_{0}, T\right]$.

Proof. Proof. Similar to Theorem 4.1.

Remark 4.2. It is easy to show that convergence order of Adams-Bashforth of fourth order is $O\left(h^{4}\right)$.

Theorem 4.3. Adams-Bashforth of fourth order is stable.

Proof. For Adams-Bashforth of fourth order exist only one characteristic polynomial $P(\lambda)=\lambda^{4}-1$ and it is clear that satisfies the root condition then by Theorem 2.1, the Adams-Bashforth of fourth order is stable.

Theorem 4.4. Milne-Simpson of fourth order is stable.

Proof. Similar to Theorem 4.3.

The reason of choosing the Adams-Bashforth of fourth order and Milne-Simpson of fourth order as a predictor-corrector technique is that both of them are stable.

\section{Numerical Examples}

Example 5.1. Consider the initial value problem

$\widetilde{y}^{\prime}(t)=-\widetilde{y}(t), 0 \leq t \leq 1$

$\widetilde{y}(0)=(0.96+0.04 \alpha, 1.01-0.01 \alpha)$

$\widetilde{y}(0.01)=(0.9504+0.0396 \alpha, 1-0.0099 \alpha)$

$\widetilde{y}(0.02)=(0.9410+0.0392 \alpha, 0.99-0.0098 \alpha)$

$\widetilde{y}(0.03)=(0.9316+0.0388 \alpha, 0.9801-0.0097 \alpha)$

The exact solution at $t=0.1$ is given by

$$
Y(0.1, \alpha)=\left((0.96+0.04 \alpha) e^{-0.1},(1.01-0.01 \alpha) e^{-0.1}\right),
$$

The results of Example 5.1 are shown in Table 1 and 2 by using the predictorcorrector method. In Figure 1, comparison between the exact solution, Adams PC Method solution and Milne's PC method solution of Example 5.1 is presented. 
Example 5.2. Consider a simple $R L$ circuit (The ' $R L$-circuit' is an abbreviation of Resistance-Inductance circuit). The ordinary differential equation corresponding to this electrical circuit is $i^{\prime}(t)=-\frac{R}{L} i(t)+v(t), t_{0} \leq t_{0}+a$, subject to the initial condition $i\left(t_{0}\right)=i^{0}$, where $R$ is the circuit resistance, $L$ is the coefficient corresponding to the solenoid, and $v$ is the voltage function. Environmental conditions, in accuracy in element modeling, electrical noise, leakage, and other parameters cause uncertainty in the aforementioned equation considering it instead as a fuzzy initial value problem yields more realistic results. This innovation helps to detect unknown conditions in circuit analysis [37],[38]. Consider an electrical $R L$ circuit with an $A C$ source: $i^{\prime}(t)=-\frac{R}{L} i(t)+v(t), 0 \leq t \leq 1$ subject to the uncertain initial condition $i(0)=u$.

Suppose that $R=1 \mathrm{Ohm}, L=1$ Henry,

$$
v(t)=\sin (t)
$$

and

$$
u(s)=\left\{\begin{array}{c}
25 s-24,0.96 \leq s<1, \\
-100 s+101,1 \leq s \leq 1.01, \\
0,
\end{array}\right.
$$

Thus, the initial value problem has

$$
\widetilde{i}^{\prime}(t)=-\widetilde{i}(t)+\sin (t)
$$

subject to the initial conditions

$\widetilde{i}(0)=(0.96+0.04 \alpha, 1.01-0.01 \alpha)$

$\widetilde{i}(0.01)=(0.9505+0.0396 \alpha, 1-0.0099 \alpha)$

$\widetilde{i}(0.02)=(0.9412+0.0392 \alpha, 0.9902-0.0098 \alpha)$

$\widetilde{i}(0.03)=(0.9321+0.0388 \alpha, 0.9806-0.0097 \alpha)$

The exact solution at $t=0.1$ is given by

$$
I(0.1, \alpha)=\left(\frac{\sin (0.1)-\cos (0.1)}{2}+\frac{1}{2} e^{-t}+e^{-t} u\right)
$$

where

$$
[u]^{\alpha}=\left[\frac{24}{25}+\frac{1}{25} \alpha, \frac{101}{100}-\frac{1}{100} \alpha\right]
$$

The results of Example 5.2 are shown in Table 3 and 4 by using the predictorcorrector method. In Figure 2, comparison between the exact solution, Adams PC Method solution and Milne's PC method solution of Example 5.2 is presented. 
TABLe 1. By using the predictor-corrector method with $N=10$ the following results are obtained for Example 5.1.

\begin{tabular}{cccccc}
\hline$\alpha$ & $\underline{Y}$ (Exact) & $y$ (Adams) & $y$ (Milne) & $\underline{y}$ (Adams Error) & $\underline{y}$ (Milne Error) \\
\hline 0 & 0.8686439 & 0.8689297 & 0.8686356 & 0.0002857 & 0.0000082 \\
0.1 & 0.8722632 & 0.8725204 & 0.8722558 & 0.0002572 & 0.0000074 \\
0.2 & 0.8758836 & 0.8761112 & 0.8758760 & 0.0002286 & 0.0000065 \\
0.3 & 0.8795019 & 0.8797020 & 0.8794962 & 0.0002000 & 0.0000057 \\
0.4 & 0.8831213 & 0.8832927 & 0.8831163 & 0.0001714 & 0.0000049 \\
0.5 & 0.8867406 & 0.8868835 & 0.8867365 & 0.0001428 & 0.0000041 \\
0.6 & 0.8903600 & 0.8904743 & 0.8903567 & 0.0001143 & 0.0000032 \\
0.7 & 0.8939793 & 0.8940651 & 0.8939769 & 0.0000857 & 0.0000024 \\
0.8 & 0.8975987 & 0.8976558 & 0.8975970 & 0.0000571 & 0.0000016 \\
0.9 & 0.9012180 & 0.9012466 & 0.9012172 & 0.0000285 & 0.0000008 \\
1 & 0.9048374 & 0.9048374 & 0.9048374 & 0.0000000 & 0.0000000 \\
\hline
\end{tabular}

TABLE 2. By using the predictor-corrector method with $N=10$ the following results are obtained for Example 5.1.

\begin{tabular}{cccccc}
\hline$\alpha$ & (Exact) & $\bar{y}$ (Adams) & $\bar{y}$ (Milne) & $\bar{y}$ (Adams Error) & $\bar{y}$ (Milne Error) \\
\hline \hline 0 & 0.9138857 & 0.9136000 & 0.9138940 & 0.0002857 & 0.0000082 \\
0.1 & 0.9129809 & 0.9127237 & 0.9129883 & 0.0002572 & 0.0000074 \\
0.2 & 0.9120761 & 0.9118474 & 0.9120827 & 0.0002286 & 0.0000065 \\
0.3 & 0.9111712 & 0.9109712 & 0.9117180 & 0.0002000 & 0.0000052 \\
0.4 & 0.9102664 & 0.9100949 & 0.9102713 & 0.0001714 & 0.0000049 \\
0.5 & 0.9093616 & 0.9092187 & 0.9093657 & 0.0001428 & 0.0000041 \\
0.6 & 0.9084567 & 0.9083424 & 0.9084600 & 0.0001143 & 0.0000032 \\
0.7 & 0.9075519 & 0.9074661 & 0.9075543 & 0.0000857 & 0.0000024 \\
0.8 & 0.9066470 & 0.9065899 & 0.9066487 & 0.0000571 & 0.0000016 \\
0.9 & 0.9057422 & 0.9057136 & 0.9057430 & 0.0000285 & 0.0000008 \\
1 & 0.9048374 & 0.9048374 & 0.9048374 & 0.0000000 & 0.0000000 \\
\hline
\end{tabular}

TABLE 3. By using the predictor-corrector method with $N=10$ the following results are obtained for Example 5.2.

\begin{tabular}{cccccc}
\hline$\alpha$ & $\underline{I}$ (Exact) & $\underline{i}$ (Adams) & $\underline{i}$ (Milne) & $\underline{i}$ (Adams Error) & $\underline{i}$ (Milne Error) \\
\hline 0 & 0.8734772 & 0.8737630 & 0.87346902 & 0.0002857 & 0.0000082 \\
0.1 & 0.8770966 & 0.8773538 & 0.87708919 & 0.0002572 & 0.0000074 \\
0.2 & 0.8807159 & 0.8809445 & 0.88070937 & 0.0002286 & 0.0000065 \\
0.3 & 0.8843353 & 0.8845353 & 0.88432954 & 0.0002000 & 0.0000057 \\
0.4 & 0.8879546 & 0.8881261 & 0.88794971 & 0.0001714 & 0.0000049 \\
0.5 & 0.8915740 & 0.8917169 & 0.89156988 & 0.0001428 & 0.0000041 \\
0.6 & 0.8951933 & 0.8953076 & 0.89519006 & 0.0001143 & 0.0000032 \\
0.7 & 0.8988127 & 0.8988984 & 0.89881023 & 0.0000857 & 0.0000024 \\
0.8 & 0.9024320 & 0.9024892 & 0.90243040 & 0.0000571 & 0.0000016 \\
0.9 & 0.9060514 & 0.9060799 & 0.90605058 & 0.0000285 & 0.0000008 \\
1 & 0.9096707 & 0.9096707 & 0.90967075 & 0.0000000 & 0.0000000 \\
\hline
\end{tabular}


TABLE 4. By using the predictor-corrector method with $N=10$ the following results are obtained for Example 5.2.

\begin{tabular}{cccccc}
\hline$\alpha$ & $\bar{I}$ (Exact) & $\bar{i}$ (Adams) & $\bar{i}$ (Milne) & $\bar{i}$ (Adams Error) & $\bar{i}$ (Milne Error) \\
\hline 0 & 0.9187191 & 0.9184333 & 0.9187273 & 0.0002857 & 0.0000082 \\
0.1 & 0.9178142 & 0.9175570 & 0.9178216 & 0.0002572 & 0.0000074 \\
0.2 & 0.9169094 & 0.9166808 & 0.9169160 & 0.0002286 & 0.0000065 \\
0.3 & 0.9160046 & 0.9158045 & 0.9160103 & 0.0002000 & 0.0000057 \\
0.4 & 0.9150997 & 0.9149283 & 0.9151047 & 0.0001714 & 0.0000049 \\
0.5 & 0.9141949 & 0.9140520 & 0.9141990 & 0.0001428 & 0.0000041 \\
0.6 & 0.9132901 & 0.9131757 & 0.9132933 & 0.0001143 & 0.0000032 \\
0.7 & 0.9123852 & 0.9122995 & 0.9123877 & 0.0000857 & 0.0000024 \\
0.8 & 0.9114804 & 0.9114232 & 0.9114820 & 0.0000571 & 0.0000016 \\
0.9 & 0.9105755 & 0.9105470 & 0.9105764 & 0.0000285 & 0.0000008 \\
1 & 0.9096707 & 0.9096707 & 0.9096707 & 0.0000000 & 0.0000000 \\
\hline
\end{tabular}

Figure 1. Comparison between the Exact (-), Adams PC Method(-) and Milne's PC method(...) of Example 5.1.

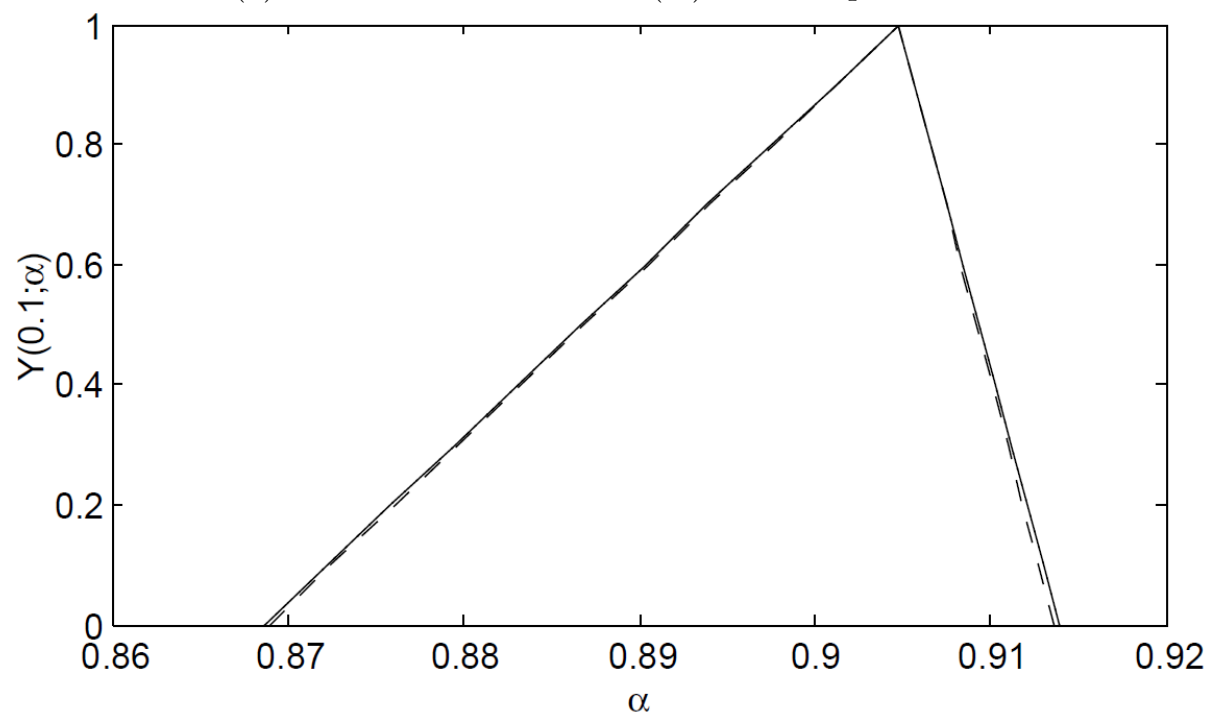


Figure 2. Comparison between the Exact (-), Adams PC Method(-) and Milne's PC method(...) of Example 5.2.

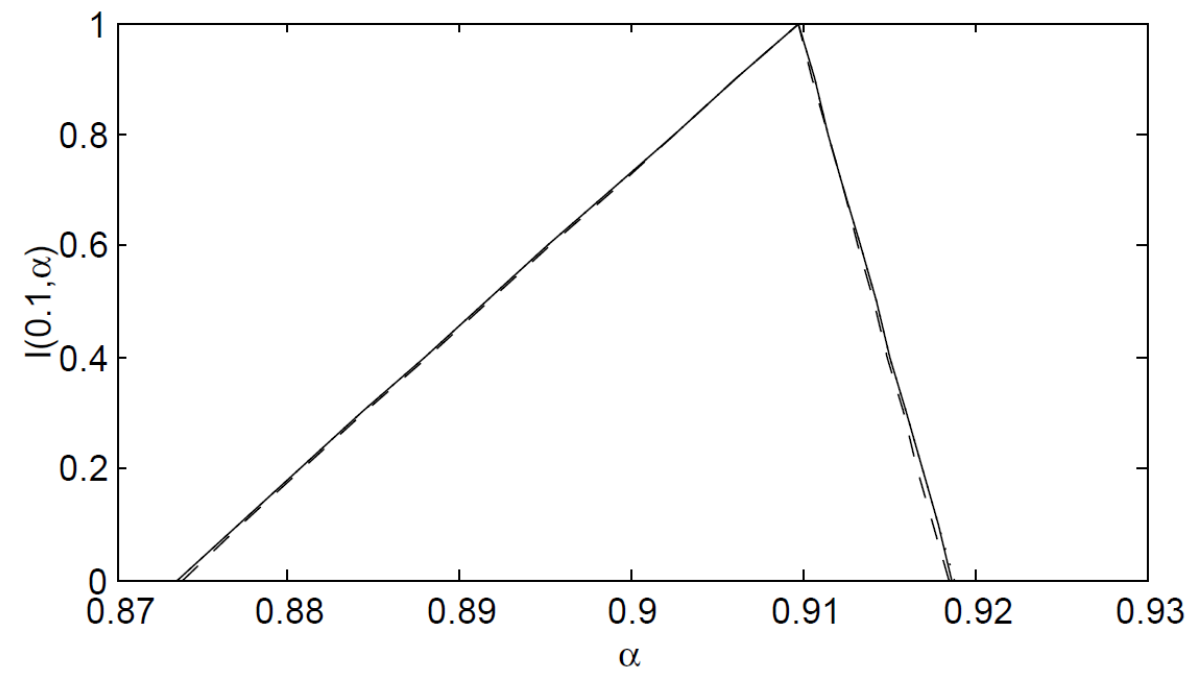

\section{Conclusions}

In this paper, the Milne's predictor corrector method is investigated for solving of fuzzy differential equation. A consistency order with two examples is proved to show the efficiency the predictor-corrector method.

\section{REFERENCES}

[1] Guo, M., Xue, X. and Li, R., Impulsive functional differential inclusions and fuzzy population models. Fuzzy Sets and Systems 138 (2003), 601-615.

[2] El Naschie, M.S., A review of $E$-infinite theory and the mass spectrum of high energy particle physics. Chaos, Solitons and Fractals 19 (2004), 209-236.

[3] El Naschie, M.S., The concepts of $E$-infinite: an elementary introduction to the Cantorianfractal theory of quantum physics. Chaos, Solitons and Fractals 22 (2004), 495-511.

[4] El Naschie, M.S., On a fuzzy Khler manifold which is consistent with the two slit experiment. International Journal of Nonlinear Science Numerical Simulation 6 (2005), 95-98.

[5] Tanaka,Y., Mizuna Y. and Kado, T., Chaotic dynamics in the Friedman equation. Chaos, Solitons and Fractals 24 (2005), 407-422.

[6] El Naschie, M.S., From experimental quantum optics to quantum gravity via a fuzzy Khler manifold. Chaos, Solitons and Fractals 25 (2005), 969-977.

[7] Zhang, H., Liao, X. and Yu, J., Fuzzy modelling and synchronization of hyperchaotic systems. Chaos, Solitons and Fractals 26 (2005), 835-843.

[8] Feng, G. and Chen, G., Adaptive control of discrete-time chaotic systems: a fuzzy control approach. Chaos, Solitons and Fractals 23 (2005), 459-467.

[9] Jiang, W., Guo-Dang, Q. and Bin, D., $H_{\infty}$ variable universe adaptive fuzzy control for chaotic systems. Chaos, Solitons and Fractals 24 (2005), 1075-1086.

[10] Abbad, M.F.,Von Keyserlingk, D.G.,Linkens, D.A. and Mahfouf,M., Survey of utilisation of fuzzy technology in medicine and healthcare. Fuzzy Sets and Systems 120 (2001), 331-349.

[11] Barro, S. and Marn, R.,Fuzzy logic in medicine,Heidelberg, Physica-verlag,2002.

[12] Helgason, C.M. and Jobe, T.H., The fuzzy cube and causal efficiacy: representation of concomitant mechanisms in stroke. Neural Networks 11 (1998), 549-555.

[13] Nieto, J.J. and Torres, A., Midpoints for fuzzy sets and their application in medicine. Artificial Intelligence in Medicine 27 (2003), 81-101. 
[14] Bandyopadhyay, S., An efficient for superfamily classification of amino acid sequences: feature extraction, fuzzy clustering and prototype selection. Fuzzy Sets and Systems 152 (2005), 5-16.

[15] Casasnovas, J. and Rossell, F., Averaging fuzzy biopolymers. Fuzzy Sets and Systems 152 (2005), 139-158.

[16] Chang, B.C. and Halgamuge, S.K., Protein motif extraction with neuro-fuzzy optimization. Bioinformatics 18 (2002), 1084-1090.

[17] Dembl, D. and Kastner, P., Fuzzy $C$-means method for clustering microarray data. Bioinformatics 19 (2003), 973-980.

[18] Heger, A. and Halm, L.,Sensitive pattern discovery with fuzzy alignments of distantly related proteins. Bioinformatics 19 (2003), 130-137.

[19] Nieto, J.J., Torres, A., Georgiou, D.N. and Karakasidis, T.E., Fuzzy polynucleatide spaces and metrics. Bulletin of Mathematical Biology 68 (2006), 301-317.

[20] Kaleva, O., Fuzzy differential inclusions. Fuzzy Sets and Systems 24 (1987), 301-317.

[21] Kloeden, P., Remarks on Peano-like theorems for fuzzy differential equations. Fuzzy Sets and Systems 44 (1991), 161-164.

[22] Seikkala, S., On the fuzzy initial value problem, Fuzzy Sets and Systems 24 (1987), 319-330.

[23] Song, S. and Wu, C., Existence and uniqueness of solution to the Cauchy problem of fuzzy differential equations. Fuzzy Sets and Systems 110 (2000), 55-67.

[24] Wu,C., Song, S. and Stanley Lee, E.,Approximate solution existence and uniqueness of the Cauchy problem of fuzzy differential equations. Journal of Mathematical Analysis and Applications 202 (1996), 629-644.

[25] Kaleva, O., The Cauchy problem for fuzzy differential equations. Fuzzy Sets and Systems 35 (1990), 389-396.

[26] He, Q. and Yi, W., On fuzzy differential equations. Fuzzy Sets and Systems 32 (1989), 321325.

[27] Menda, W., Linear fuzzy differential equation system on $\mathbb{R}^{1}$. Journal of Fuzzy Syst Math 2(1) (1988), 51-56 (in Chinese).

[28] Allahviranloo, T., Ahmadi,N. and Ahmadi, E., Numerical solution of fuzzy differential equations by predictor-corrector method. Information sciences 177 (2007), 1633-1647.

[29] Friedman,M., Ma, M. and Kandel, A., Numerical solution of fuzzy differential and integral equations. Fuzzy Sets and Systems 106 (1999), 35-45.

[30] Ma, M., Friedman, M. and Kandel, A., Numerical solution of fuzzy differential equations. Fuzzy Sets and Systems 105 (1999), 133-138.

[31] Khastan, A. and Ivaz, K., Numerical solution of fuzzy differential equations by Nyström method. Chaos, Solitons and Fractals 41 (2009), 859-865.

[32] Isaacson, E. and Keller, H.B.,Analysis of Numerical Methods, Wiley, New York, 1966.

[33] Stefanini, L.,Sorini, L. and Guerra, M.L., Parametric representation of fuzzy numbers and application to fuzzy calculus. Fuzzy Sets and Systems 157 (2006),2423-2455.

[34] Dong-Kai, Z., Wen-Li, F., Ji-qing, Q. and Duoming, X., On the study of linear properties for fuzzy number-valued fuzzy integrals. Fuzzy Information and Engineering 54 (2009),227-232.

[35] Colombo, G. and Krivan, V.,Fuzzy differential inclusions and non-probabilistic likelihood. Dynamic Systems and Applications 1 (1992),419-440.

[36] Kaleva, O.,Interpolation of fuzzy data. Fuzzy Sets and Systems 35 (1990),389-396.

[37] Effati, S. and Pakdaman, M., Artificial neural network approach for solving fuzzy differential equations. Information Sciences 180 (2010),1434-1457.

[38] Abu-Argub, O., El-Ajou, A., Momani, S.and Shawagfeh, N.,Analytical solutions of fuzzy initial value problems by HAM. Applied Mathematics and Information Sciences 5 (2013),19031909.

Department of Mathematics, Kocaeli University, Izmit, Kocaeli,Turkey

E-mail address: aylin@kocaeli.edu.tr 